TEMAS CRITICOS

\title{
Desafíos interdisciplinarios en los estudios críticos del desarrollo
}

\begin{abstract}
Yolanda Alfaro"
Para alcanzar la interdisciplinariedad en el campo de estudios críticos del desarrollo no sólo es necesario superar el proceso de deducción lógica y el predominio de la economía convencional, sino que también es preciso apropiarse de formas de pensar y de construir objetos de estudio que superen las fronteras y jerarquías disciplinares, el antagonismo entre

estructura y sujeto, las disputas entre generalización y particularismo, las disparidades de los enfoques cualitativos y cuantitativos y la desarticulación entre teoría y práctica.
\end{abstract}

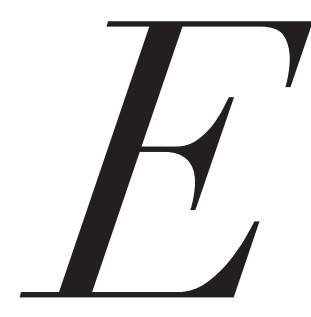

n América Latina, a partir de la década del noventa, los estudios críticos del desarrollo se han configurado como un campo de investigación y debate sobre los patrones o paradigmas de desarrollo que pretendieron legitimar la instrumentación del programa de reformas estructurales del Banco Mundial (Bм) y el Fondo Monetario Internacional (FMI), y con ello la práctica de gobiernos, organismos internacionales, organizaciones no gubernamentales y algunos sectores de la sociedad civil y la academia.

*Estudiante del Doctorado en Estudios del Desarrollo de la Universidad Autónoma de Zacatecas, México.
Para llevar a cabo tal propuesta fue necesario, por una parte, repensar el enfoque epistemológico que fundamentaba la visión del desarrollo en las ciencias sociales, puesto que en el contexto neoliberal las academias y sus concepciones teórico-metodológicas fueron uno de los medios para insertar las perspectivas de desarrollo humano, alternativo, local, sustentable, etc. Y, por otra parte, las prácticas de desarrollo que, en contextos específicos, los distintos actores impulsan como estrategias para confrontar los cambios políticos, económicos y culturales. Actualmente, este reto se convirtió en el fundamento de los estudios críticos del desarrollo; pues su carácter crítico no sólo propone producir conocimientos teóricos con base en una perspectiva 
alternativa, sino aportar herramientas prácticas para orientar los procesos de transformación social en curso o aquellos que se están gestando.

De cara a este desafío se plantearon una serie de propuestas que postulan la necesidad de: 1) reconocer que el desarrollo en sus diversas dimensiones es heterogéneo e involucra a distintos actores en su práctica, y 2) adoptar un método de investigación acorde con la naturaleza multidimensional del objeto de estudio y la condición estructural o estratégica del proceso de desarrollo, de manera que la articulación entre teorías, conceptos y evidencia empírica sea una condición sine qua non del oficio y la construcción (idea y práctica) del desarrollo.

En el campo de estudios críticos del desarrollo el desafío de la interdisciplinariedad no sólo implicó abrir diálogos entre diversos puntos de vista disciplinares con el fin de alcanzar una comprensión profunda del tema, sino también recuperar los principios epistemológicos como el elemento de la argumentación teórica, metodológica y práctica esencial para el desarrollo. A pesar de los esfuerzos realizados, este desafío todavía no se ha logrado alcanzar en la práctica de la investigación, precisamente porque demanda despojarse de la estructura del proceso de deducción lógica que ha permeado el campo de estudios del desarrollo bajo el predominio de la economía convencional, y en su lugar apropiarse de formas de pensar y de construir objetos de estudio que superen: i) las fronteras y jerarquías disciplinares que lo determinan; ii) el antagonismo entre la determinación de la estructura y la praxis del sujeto; iii) las disputas entre lo general y lo particular; iv) las disparidades de los enfoques cualitativos y cuantitativos, $\mathrm{y} v$ ) la desarticulación entre la teoría y la práctica. Se trata de construir un método que sea deductivo e inductivo a la vez.

\section{CAMINO RECORRIDO: INTERDISCIPLINARIEDAD EN LOS ESTUDIOS DEL DESARROLLO}

En América Latina las discusiones sobre la interdisciplinariedad y su aplicación en el desarrollo no se dieron a la par de los países desarrollados; esto se debió a que las tradiciones positivistas desarrolladas en Europa y Estados Unidos, tales como el empirismo lógico, fueron cuestionadas por las teorías de la dependencia en su corriente marxista y weberiana, pero tuvieron muy poca repercusión debido a que en la década de los ochenta el neoliberalismo y sus reformas estructurales delinearon los debates académicos desde las escuelas de economía convencional o neoclásica, truncando así los intentos de la teoría de la dependencia y el marxismo por construir alternativas metodológicas para el análisis del desarrollo que se sustenten en la visión histórico-estructural. ${ }^{1}$

Los estudios del desarrollo desde la economía, la sociología política y las relaciones internacionales centraron sus intereses de investigación en tratar de dilucidar el crecimiento económico y los obstáculos internos de los países subdesarrollados para impulsar su crecimiento-desarrollo. En este periodo predominó la visión etapista y lineal del desarrollo, fundamentalmente afiliadas a dos corrientes de pensamiento: $i$ ) la escuela neoclásica que planteaba que el desarrollo es un aspecto estrictamente económico que debe enfocarse en los factores que construyen un mercado en equilibrio permanente, y ii) la corriente keynesiana que planteaba el desarrollo como la constante expansión de la demanda efectiva mediante estímulos gubernamentales.

Ambas corrientes teóricas tuvieron como peculiaridad - con variantes menores - la construcción de un conjunto de instrumentos para analizar los problemas esenciales del desarrollo: el crecimiento del valor de la producción económica, el crecimiento del Producto interno bruto (PIB), el desempleo estructural, etc. Metodológicamente, estas propuestas invirtieron el proceso de construcción del conocimiento en tanto que redujeron la realidad económica a la lógica del razonamiento economicista, ${ }^{2}$ motivo por el cual resultaron insuficientes a la hora de construir una explicación científica del proceso social de producción, circulación, distribución e intercambio que conlleva el desarrollo económico.

En la década de los ochenta, con el advenimiento del neoliberalismo, el estudio del desarrollo se enfocó en los problemas que traía consigo la inserción de las economías nacionales en la globalización, la competitividad y el funcionamiento del mercado autorregulado y, con ello, la condición dependiente y subdesarrollada de los países del Sur global. La teoría de la dependencia planteó un primer intento de ruptura con la visión economicista del desarrollo demostrando empíricamente que el crecimiento podía producirse sin consecuencias sociales positivas para la sociedad. De hecho, para los dependentistas, el concepto de desarrollo visto desde los neoclásicos y keynesia- 
nos carecía de sustento científico porque no lo comprendían como "proceso histórico", como una dinámica que transcurre bajo determinadas condiciones históricas. Este giro implicó una nueva definición de desarrollo, más cercana al mejoramiento del bienestar de la población, dado que para dar cuenta del nivel de desarrollo que habían alcanzado las sociedades era necesario recurrir a las características, económicas, tecnológicas y demográficas, y a las condiciones históricas y políticas de cada una.

A mediados de la década de los ochenta, los estudios del desarrollo una vez más adquirieron nuevos horizontes; no obstante, en el pensamiento social latinoamericano, más que predominar el neoliberalismo o sus versiones ampliadas de la teoría de la elección racional, prevaleció un panorama de dispersión teórica y metodológica. Desde disciplinas como la antropología y la historia se rebatieron las condiciones de determinismo de las fuerzas que impulsan el desarrollo, planteando la perspectiva basada en los actores sociales, cuyo argumento central se asentaba en la visión estratégica del desarrollo, es decir, en el reconocimiento de que son los propios pueblos, sobre la base de acciones participativas y principios de organización autónoma, los que construyen sus propios procesos de desarrollo.

Con esta nueva perspectiva se introdujo en la agenda de debate la dimensión espacio-territorial de los procesos de desarrollo y el lugar de los sujetos como actores del desarrollo local, de manera que los problemas claves del desarrollo se enmarcaron en el capital social, las interfaces de conocimiento, redes y poder, las interrelaciones de la globalización y el localismo, así como las dinámicas del discurso de la política y la intervención planificada.

Los paradigmas de la agency, las teorías de la acción comunicativa, la de sistemas, la de elección racional, el postestructuralismo, los estudios feministas poscoloniales, entre otros, no sólo reconfiguraron los debates en torno a la escala del desarrollo sino que incorporaron una nueva forma de investigación empírica al plantear el análisis de la construcción social del desarrollo en oposición a los análisis estructurales, institucionales y de economía política. Los estudios de caso con métodos etnográficos y la investigación-acción participativa que se desarrollaron en la antropología influyeron de manera muy importante en este periodo, puesto que sirvieron de referente para quienes pretendían explicar el desarrollo desde la agencia de los actores locales y los procesos de cambio social que se impulsan desde abajo y de manera endógena. ${ }^{3}$

A mediados de la década de los noventa, este enfoque fue adoptado por los gobiernos en pos de instrumentar políticas de desarrollo con perspectiva local, mismas que fueron ampliamente respaldadas por los organismos internacionales y el mandato del Posconsenso de Washington: participación popular, empoderamiento de los pobres, descentralización del gobierno nacional, entre otras tantas. De esta manera, la teoría orientada al actor, sus conceptos y métodos fueron funcionalizados a los principios y objetivos del modelo de desarrollo neoliberal, lo cual desvirtuó el potencial explicativo de la perspectiva centrada en los temas relacionados con la concepción de las agencias y los procesos de cambio social.

En los últimos 10 años, el pensamiento latinoamericano, especialmente de los países con gobiernos progresistas, ha vuelto a plantear una nueva perspectiva para el estudio del desarrollo bajo el llamado Buen Vivir o Vivir Bien. El eje articulador de estas propuestas ha sido la necesidad de repensar los supuestos del modelo de desarrollo imperante a partir de premisas radicalmente distintas a las convencionales; esto quiere decir poner en cuestión las (meta) teorías y los conceptos con que se ha interpretado la búsqueda de mejores condiciones de vida y las tendencias sociales, económicas y culturales para conseguirlas desde finales de la segunda guerra mundial mediante tres supuestos: $i$ ) el antropocentrismo con que se ha llevado a cabo el proyecto de la modernidad; ii) la linealidad del progreso, y iii) la racionalidad de los parámetros con que se ha medido el desarrollo

Las últimas décadas han estado marcadas por la necesidad de deslegitimar la explicación e interpretación del desarrollo desde las condiciones estructurales o desde la agencia del actor, según sea la posición política que asuman los investigadores; no obstante, el afán por demostrar las diferencias en los métodos de investigación y el significado teórico atribuido a la idea y práctica del desarrollo, desde uno u otro enfoque, no han logrado más que plantear posturas de moral académica que impiden romper con el razonamiento disciplinar y unidimensional del desarrollo que se ilustra en la tendencia generalizada de medir el desarrollo en términos de crecimiento económico.

Debatir sobre las estructuras conceptuales y las acepciones epistemológicas, particularmente sobre cómo abordar metodológicamente el desarrollo, es un reto 
fundamental para los estudios críticos del desarrollo. El centro de la preocupación radica en re-elaborar estrategias analíticas que permitan un diálogo interdisciplinario capaz de abordar el objeto de estudio desde un enfoque teórico-metodológico capaz de responden a la multidimencionalidad de los procesos, su contingencia, la diversidad de actores y las interconexiones con los procesos de transformación contemporáneos.

La interdisciplinariedad en los estudios críticos del desarrollo, por una parte, implica la toma de conciencia de los límites disciplinares y las determinaciones teóricas a priori, así como asumir críticamente las contradicciones metodológicas que se han creado entre los enfoques cuantitativo y cualitativo. Cabe advertir que la interdisciplinariedad es una condición que requiere de un amplio sentido crítico y una actitud de tolerancia para entender la realidad desde distintos puntos de vista, sin que ello signifique perder de vista el posicionamiento político que debe tener la investigación social.

Por otra parte, la interdisciplinariedad en los estudios críticos del desarrollo implica el reconocimiento de que su objeto no es específico de una sola disciplina, sino que al tratarse de un campo tan específico y amplio, el objeto de estudio es de singular complejidad, por lo que su investigación requiere de un conjunto de contenidos disciplinares puntuales y del diseño de estrategias de investigación con metodologías que combinen técnicas cualitativas y cuantitativas.

No obstante, cabe preguntarse ¿cuáles son los principios que pueden o podrían encausan la interdisciplinariedad en los estudios críticos del desarrollo?, ¿a partir de qué principios se puede construir un marco de investigación común en el campo y una forma particular de acercamiento al objeto de estudio en las ciencias sociales?

\section{UNA ALTERNATIVA METODOLÓGICA PARA LOS ESTUDIOS DEL DESARROLLO}

Para que la interdisciplinariedad sea una alternativa metodológica para los estudios del desarrollo debemos partir reconociendo que no se trata de la simple sumatoria de disciplinas sino de una nueva concepción epistemológica de la realidad, por lo tanto, se requiere trabajar en la construcción de una estructura analítica que permita realizar lecturas integradas del problema en cuestión, esto en virtud de responder no sólo a los pa- trones de la investigación científica (validación empírica), sino de construir verdaderas respuestas sociales.

El método interdisciplinar ${ }^{4}$ en los estudios del desarrollo estaría constituido por cuatro aspectos centrales: semántico (visión crítica del desarrollo), pragmático (aplicación a la resolución de problemas), sociológico (aceptado por una comunidad) e histórico (con validez temporal). Estas consideraciones suponen fundamentar la interdisciplinariedad como enfoques deductivo e inductivo a la vez. Desde la historicidad, los límites disciplinarios son mucho más difusos por lo que es preciso diseñar el problema de investigación en diferentes niveles de análisis y en correspondencia con una estrategia de multimétodos, es decir, métodos de corte cuantitativos como cualitativos, de manera que los hallazgos de la investigación sean producto de un diálogo entre varias disciplinas. "La historicidad cumple la función de permitirnos reconocer la articulación del fenómeno, ubicado en un determinado contexto, sea porque es articulador, sea porque es capaz de ser articulado." ${ }_{5}$

Este enfoque plantea dos interesantes retos para el campo de estudios críticos del desarrollo: uno subyacente a los problemas del desarrollo y su conceptualización y, otro, en relación a sus dinamismos constitutivos que se refieren a la forma en que se construyen, reproducen y transforman en el tiempo los problemas inherentes al desarrollo como idea y práctica.

En ese sentido, la interdisciplinariedad como método de investigación propio de los estudios del desarrollo se basaría en la articulación de los distintos planos de la realidad:

a) articulación del espaciamiento económico, político, social, etc.;

b) articulación espacio-temporal como las vinculaciones macro-micro, periodo-coyuntura, secuencia-momento, proceso-estructura, y

c) articulación de la dialéctica praxis-estructura, sujeto activo-fuerzas sociales, sujeto insurgenteconciencia política.

La articulación interdisciplinaria plantea una ruptura con la visión estática de teoría - regida por una lógica determinada en conceptos y teorías previamente establecidas - , supone la reconstrucción crítica basada en la forma específica en que los diversos planos de la realidad se articulan en un espacio y tiempo determinados y pretende construir una relación teórica y práctica de las características y las pro- 
piedades de un objeto de estudio. ${ }^{6}$ El propósito de la articulación interdisciplinaria en el campo de los estudios críticos del desarrollo consiste en una problematización del objeto de estudio desde sus diversas perspectivas de modo tal que permita definir un campo de opciones que trascienda las disciplinas.?

Esta propuesta está estrechamente relacionada con cuestiones epistemológicas de la relación entre teoría y práctica, puesto que para construir parámetros de análisis interdisciplinar se requiere establecer conexiones y mediaciones teóricas y metodológicas que rompan con la tendencia dominante en el plano de la investigación científica. De hecho, problematizar el desarrollo desde sus múltiples acepciones, condiciones y dimensiones mediante la aplicación de metodologías cuantitativas y cualitativas constituye un punto particularmente importante en el campo, porque cada uno provee información que no sólo es diferente a la dada por el otro, sino que, además, es esencial para interpretar a la otra. ${ }^{8}$

La llamada convergencia metodológica o triangulación, que Denzin planteó ya en la década de los setenta, puede ser un camino para romper con la supuesta contradicción entre uno y otro método, en vista de que plantea el uso de múltiples disciplinas para la creación de la teoría; no obstante, para lograr una perspectiva interdisciplinaria en el campo de los estudios críticos del desarrollo es necesario adoptar la integración y la articulación como los supuestos epistemológicos del análisis, de manera que los problemas que subyacen al tema del desarrollo puedan ser interpretados desde diferentes tipos de datos pero en función de un razonamiento interdisciplinar. Esto es de vital importancia porque la articulación no se refiere exclusivamente a las disciplinas, sino a la articulación de varios planos de la realidad social - la naturaleza de su dinámica y la especificidad de su comportamiento y orientación-, ya que permite descubrir de manera empírica relaciones posibles o replantear algunas anticipadas teóricamente.

\section{Hacia la CONSTRUCCión}

\section{DE LA INTERDISCIPLINARIEDAD}

Los planteamientos teóricos de la interdisciplinariedad $^{9}$ resultan muy tentadores para debatir sobre la posibilidad de utilizar la integración - teoría-práctica - y la articulación - espacio temporal; praxis-estructuracomo principios metodológicos para el campo de los estudios del desarrollo en la medida que, por una parte, constituye una posición crítica frente a las limitaciones epistemológicas de las teorías dominantes del desarrollo y, por otra, en tanto recurso del razonamiento crítico, propone la función constructiva del conocimiento para repensar críticamente las teorías, modelos, paradigmas, programas, ideas y utopías asociadas a los estudios del desarrollo y avanzar hacia nuevas configuraciones político-teóricas y, así, dar respuestas plausibles a los cambios económicos, políticos, sociales y culturales del desarrollo. No obstante, para asumir el verdadero sentido crítico (ético) de la interdisciplinariedad es necesario reconocer sus potencialidades y limitaciones de la práctica de investigación para evitar caer en el eclecticismo o en el empantanamiento de la idealización, al punto de hacer del campo algo inútil.

\section{$-\operatorname{notas} \cdot-$}

\footnotetext{
${ }^{1}$ Enrique de la Garza Toledo (2002), "La configuración como alternativa al concepto estándar de teoría”, en Guadalupe Valencia y Enrique de la Garza y Hugo Zemelman (coords.), Epistemología y sujetos: algunas contribuciones al debate, México, спсн-unam-Plaza y Valdés.

${ }^{2}$ Tal como señala Orjuela $(2012)$.

${ }^{3}$ Norman Long (2007), Sociología del desarrollo: una perspectiva centrada en el actor, México, El Colegio de San Luis/ciesas.

${ }^{4}$ Zemelman (1989), Crítica epistemológica de los indicadores, México, El Colegio de México.
}

\footnotetext{
ZZemelman (2002), "Conciencia de la realidad y voluntad de conocer: a manera de prólogo", en G. Valencia, E. de la Garza y H. Zemelman (coords. op. cit., p. 12 .

${ }^{6}$ De la Garza (2002), op. cit.

'Zemelman, (1989), op. cit.

${ }^{8}$ Irene Vasilachis (1993), Métodos cualitativos. Los problemas teóricos epistemológicos, Buenos Aires, Editorial de América Latina.

${ }^{9}$ Zemelman (1989), op. cit.
} 
SEMINAP

\section{SOBRES}
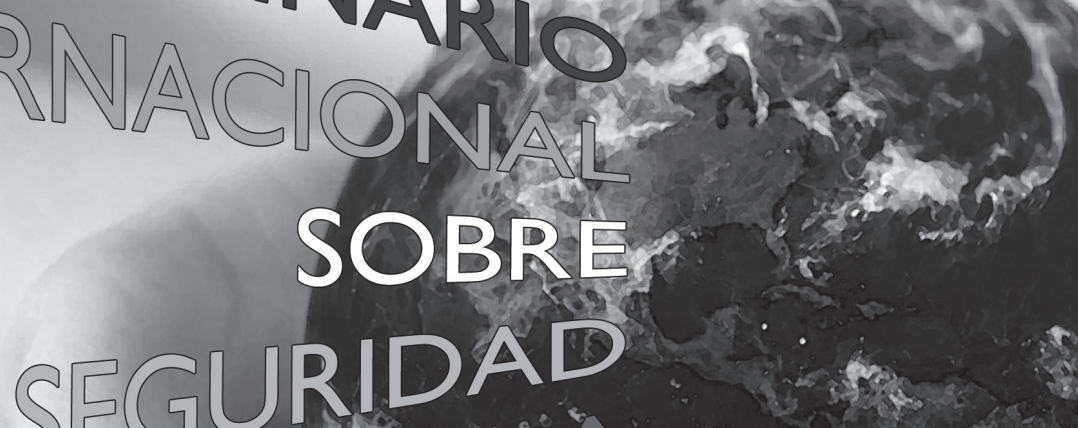

SEGURIDANA

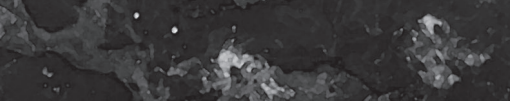

mis
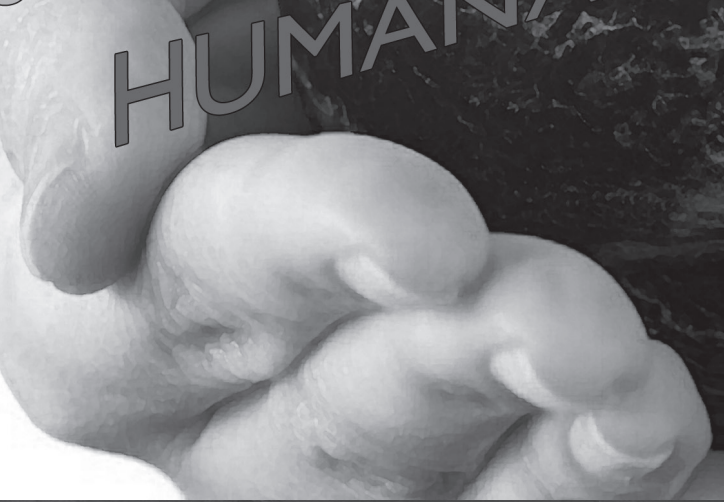

\section{I noviembre2013 II:00- | 4:30h}

Auditorio del Doctorado en Estudios del Desarrollo

\section{CON LA PARTICIPACIÓN DE}

Michael Humprey Universidad Sydney

Esthela Valverde Universidad Macquarie

Rodolfo García Zamora Universidad Autónoma de Zacatecas

Raúl Delgado Wise Universidad Autónoma de Zacatecas

Humberto Márquez Covarrubias Universidad Autónoma de Zacatecas

Guadalupe González Hernández Universidad Autónoma de Zacatecas 